\title{
ILCEA
}

Revue de l'Institut des langues et cultures

d'Europe, Amérique, Afrique, Asie et Australie

$24 \mid 2015$

Lire et écrire ensemble

\section{La poésie chorégraphique de Susana Thénon}

The Choreographic Poetry of Susana Thénon

La poesía coreográfica de Susana Thénon

Juanita Cifuentes-Louault

\section{(2) OpenEdition}

Journals

Édition électronique

URL : http://journals.openedition.org/ilcea/3577

DOI : 10.4000/ilcea.3577

ISSN : 2101-0609

Éditeur

UGA Éditions/Université Grenoble Alpes

Édition imprimée

ISBN : 978-2-84310-313-1

ISSN : 1639-6073

Référence électronique

Juanita Cifuentes-Louault, « La poésie chorégraphique de Susana Thénon », ILCEA [En ligne], 24 | 2015, mis en ligne le 21 octobre 2015, consulté le 01 mai 2019. URL : http://journals.openedition.org/ ilcea/3577 ; DOI : 10.4000/ilcea.3577

Ce document a été généré automatiquement le 1 mai 2019.

(C) ILCEA 


\title{
La poésie chorégraphique de Susana Thénon
}

\author{
The Choreographic Poetry of Susana Thénon \\ La poesía coreográfica de Susana Thénon
}

Juanita Cifuentes-Louault

1 L'œuvre de Susana Thénon (1937-1990) constitue un ensemble fécond et pluriel, comme le confirme la publication de cinq recueils poétiques - Edad sin tregua (1958), Habitante de la nada (1959), De lugares extraños (1967), distancias (1984), Ova completa (1987) - et d'un ensemble de poèmes publiés à titre posthume et réunis dans le deuxième tome de $L a$ Morada imposible (2011). La traduction, la rédaction d'essais, et la photographie constituent d'autres activités qui témoignent de la diversité de son œuvre ${ }^{1}$.

2 On peut distinguer trois phases majeures de la création thénonienne : une période lyrique porteuse des germes d'un futur éclatement du langage poétique qui concerne les trois premiers recueils (Di Ció, 2003); une deuxième phase (1970-1982) lors de laquelle la poésie, la photographie et la danse s'entrecroisent; et une dernière phase de déconstruction et d'éclatement du langage poétique marquée par la publication des recueils distancias (début de l'écriture 1968, publication en 1984) et Ova completa (1987), où elle développe l'ironie et l'humour noir à travers un langage poétique hybride.

3 Partant de l'analyse selon laquelle la voix thénonienne trouve un espace privilégié de renouvellement et de métamorphose du processus de création à travers la traversée et le dépassement des frontières artistiques (Cifuentes, 2010), je m'intéresserai ici à la manière dont la poésie de Thénon entre en relation avec la danse ainsi qu'à l'évolution de ce rapport intersémiotique ${ }^{2}$.

4 Les interactions entre danse et poésie ont suscité l'intérêt de plusieurs auteurs sur lesquels s'appuiera notre travail (Valéry, 1957 ; Maulpoix, 1998 ; Nietzsche, 1903). Les réflexions de Michèle Fink (1992) sur le lien entre ces deux arts sont également particulièrement éclairantes. Elle signale très justement l'évolution de l'alliance danse/ sacré vers un rapport corps/forme. La modernité marquerait ainsi un moment clé qui imposerait une nouvelle interaction entre la poésie et la danse. Elle se demande par 
ailleurs en quoi la danse peut apparaître, pour la poésie, comme « une sorte d'Art-Mère qui redonne sens et substance aux mots » et avance l'hypothèse selon laquelle, «si la poésie moderne pressent que la danse peut être une voie vers le sens, c'est que la quête poétique a désormais pour pierre angulaire une interrogation du corps ». Elle souligne enfin que la danse contemporaine s'est développée à partir de « l'arrachement du corps à toute servitude et à toute convention formelle ». La place centrale du corps opérerait ainsi comme matériau commun à la poésie et à la danse.

Dans l'itinéraire poétique de Thénon, on constate des points de contact entre la danse et la poésie dès les premiers recueils. Il est donc intéressant d'explorer la singularité de cette relation. Quelle serait la nature de ce lien? Serait-il du domaine thématique, symbolique, métaphorique? Ou bien pourrait-on parler d'une incarnation de la danse dans la poésie de Thénon? Quel est le processus de circulation entre la danse et la poésie ? Quel serait le sens d'une interaction entre la poésie et la danse ? Quelles sont les manifestations textuelles de la transgression des frontières artistiques?

Dans un premier temps, je montre comment, dans la première phase poétique de l'écriture thénonienne (1958-1967), la poésie et la danse entrent en contact puis comment s'intensifie la présence du corps. L'écriture de Susana Thénon peut ainsi être analysée en tant que poésie sur la danse. Puis j'analyse dans une seconde partie les procédés par lesquels la poésie entre en danse, en soulignant les entrelacs entre les deux arts.

\title{
Tangences et superpositions : une poésie sur la danse
}

\author{
El cuerpo, \\ es nada más que todo \\ (Thénon, 2011b : 25).
}

7 La première connexion entre la poésie de Thénon et la danse semble être d'ordre thématique et métaphorique. Un échantillon de six poèmes datés de 1958 à 1967 confirme la progressive infiltration de la danse dans l'univers poétique thénonien :

- Deux poèmes publiés en 1958 dans Edad sin tregua : « Tango » et « Danza ».

- Un poème inédit et non titré, appartenant à la même période de création (daté du 5 février 1958).

- Un poème publié en 1959 dans Habitante de la nada : «El bailarín ».

- Le premier poème du manuscrit "Amor ave prohibida", écrit en 1966 puis publié de manière posthume dans le deuxième tome de La Morada imposible.

- Un poème non titré publié en 1967, issu du recueil De lugares extraños : « Dame la libertad... »

Ces six poèmes constituent autant de contacts entre la poésie thénonienne et la danse. Le premier, intitulé « Tango », s'ouvre sur la précision suivante : « Ante un cuadro de Leonor Vassena $»^{3}$. L'abolition des frontières artistiques est déjà manifeste : ce poème naît de la forte impression produite sur Thénon par un tableau de Vassena. Mais il s'articule autour de l'esthétique du mouvement et de la danse. Le poème se construit autour de la dichotomie entre inertie et mouvement. L'onomatopée "Trac» peut renvoyer à une brisure, à un crépitement ou bien au bruit des pieds qui se déplacent sur le sol. Cette densité sonore associée au "sang» contribue à souligner la vitalité et la fougue qu'insuffle la danse au corps. Le mouvement peut devenir délire, comme le souligne le participe passé employé comme adjectif «alucinada». L'immobilité («quietud»; « espera») s'oppose à l'énergie dynamique («inquietud»; «movimiento»). Les 
antonymes cristallisent l'esthétique de la tension, caractéristique de l'écriture thénonienne. Mais ils révèlent également que la danse - en tant que circulation, trajectoire et déplacement - permet de faire coexister des forces contraires :

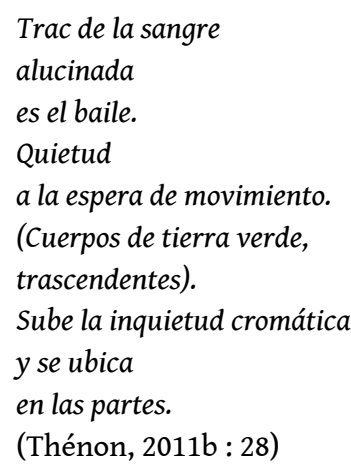

9 La voix poétique est ici témoin ou protagoniste de cette danse capable de métamorphoser les corps à son contact. Ce n'est pas un hasard si le mot « mouvement » clôt le vers central du poème et en constitue ainsi son noyau. La dimension terrestre et concrète des corps s'allie à un mouvement d'élévation: la mise entre parenthèses du vers «(Cuerpos de tierra verde, / trascendentes) » marque graphiquement un arrêt sur cette image qui allie la dimension terrestre et matérielle des corps à la métamorphose visuelle et chromatique qu'ils subissent. Comme si grâce à la danse les corps pouvaient renaître: quitter la matrice première (la terre) pour accéder à une palette beaucoup plus ample. Le chromatisme est progressif puisque la couleur ne surgit pas de manière abrupte, mais avec précision et mesure ("verdes", "se ubica»). Cela est à mettre en rapport avec le titre, « Tango ", car cette danse est très codifiée, exacte et rationnelle, contrairement à l'image véhiculée par les clichés qui l'associent à la fougue de la passion. Ce poème tisse un réseau entre la musique, la danse, la peinture et la poésie. Le dépassement lui permet d'explorer un horizon expressif beaucoup plus ample.

Le poème «Danza ", issu du même recueil, repousse plus loin encore les frontières entre les arts. Le titre même réduit la distance entre la danse et la poésie. Les pieds du danseur sont ainsi métamorphosés en plume de poète :

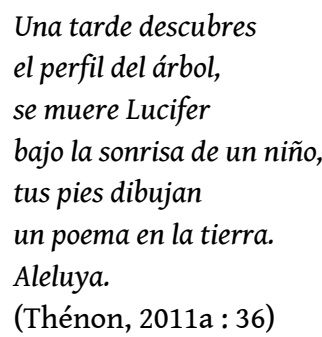

11 Les deux heptasyllabes (premier et avant-dernier vers) créent un effet ponctuel de régularité. Cependant la polymétrie globale confère au poème une élasticité rythmique signalant une tension entre la force apollinienne (ordonnée, constante, mesurée) et une dynamique dionysiaque (impulsive et impétueuse). La mention de l'arbre et de la terre souligne cette vitalité qui détruit le mal: «se muere Lucifer». Mais cette allusion au diable renvoie discrètement à l'épisode décrit dans « Le chant de la danse » de Nietzsche (1903 : 150-154), où Zarathoustra rencontre de jeunes danseuses au milieu d'une forêt. Il se présente comme "l'avocat de Dieu devant le diable» et précise que ce dernier est «l'esprit de la lourdeur ». On peut rapprocher le poème de Thénon de la philosophie nietzschéenne, dans la mesure où la légèreté et l'insouciance de la danse - symbolisées 
par le sourire d'un enfant - produisent l'anéantissement de la pesanteur et de la gravité ("se muere Lucifer»). L'exclamation finale manifeste la joie et l'extase que produit la danse comme élan et puissance libératrice. Les allitérations en [p] et en [s] transcrivent le rythme des pas sur la terre, de même que la sensualité de la danse. On retrouve dans ce poème le geste créateur comme contact du corps avec une surface qui en accueille la trace. Le danseur marque le sol tel un poète qui « avance dans la langue avec la main, en faisant aller et grincer la plume sur le papier » (Maulpoix, 1998). Ici le poème naît du contact des pieds avec la terre. Progressivement, poésie et danse entrent dans un rapport spéculaire. Le sujet poétique entre en contact et dit le monde grâce à cette « dansécriture ».

Dans les premiers poèmes faisant référence à la danse, celle-ci connecte le sujet avec la nature ${ }^{4}$. Le sujet poétique est sensible au changement des saisons, qui sont présentés comme mouvement et danse. Tel est le cas dans le poème suivant, non titré, écrit en 1958:

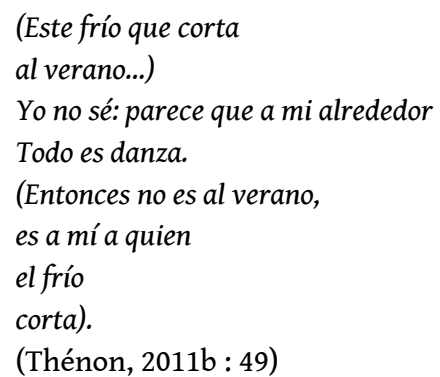

13 La place du mot «danza » à la fin du vers central est significative. Par ailleurs, un même mot («corta ») ferme le premier et le dernier vers. L'emploi de ce verbe - qui dénote la scission, la rupture - et la structure circulaire du poème créent un effet de tension. Au sein du poème coexistent des forces contraires qui favorise le mouvement, «l'éternel retour » du même. L'été du deuxième vers est remplacé par le «je » poétique, en phase avec la nature et ses mouvements. Ainsi la danse est métamorphose libératrice, comme le souligne la disposition du poème, puisque seuls les vers se référant au mouvement ne sont pas délimités par les parenthèses («todo es danza »). Or, le premier mouvement qui permet le changement, c'est le pas. Le pas de danse enclenche le déplacement, la sortie d'un espace délimité ici graphiquement représenté par les parenthèses. Faire un pas c'est éclore, changer, avancer. Dans cette perspective, un vers extrait de l'inédit «Amor Ave Prohibida » fonctionne à mon sens comme véritable art poétique :

un solo paso todo movimiento (Thénon, 2011b : 62).

Il s'agit d'un vers très simple en apparence, uniquement composé de syntagmes nominaux. Néanmoins, l'absence de verbe ne signifie pas l'inertie ou un manque d'action. $\mathrm{Au}$ contraire, elle ne fait que condenser la puissance dynamique qu'un seul pas, qu'un seul vers peut contenir. Le contraste entre l'article indéfini "un» (associé à l'adjectif soulignant sa spécificité « solo ») et l'adjectif renforçant l'idée de complétude cristallise la puissance du détail. Le pas, dans sa simplicité et sa solitude, peut engendrer une quantité incalculable de changements. En danse, un pas permet de changer de trajectoire, de direction, de rythme. Il me semble que Thénon transpose cette essence du pas au niveau poétique. Les interstices et les injections de vide introduits dans ses poèmes à la fin des années soixante fonctionneraient comme des sauts, qui, en ajustant ou en désajustant le rythme, connectent le sujet poétique à sa précarité et à son instabilité essentielle. 

l'importance des formules impératives à travers lesquelles s'exprime l'urgence de sa délivrance :

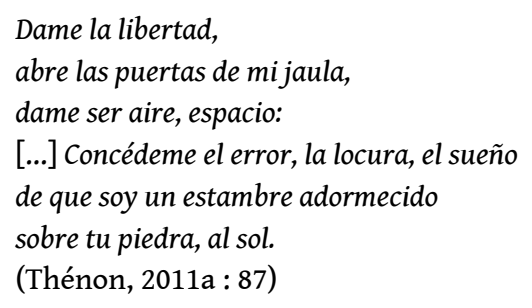

verbes à la deuxième personne de l'impératif et les deux formes enclitiques soulignent le caractère vital de la libération et de l'union avec l'autre pour le sujet poétique. Le réseau sémantique de l'espace, de la liberté et de l'affranchissement ("libertad», « espacio», «locura», sueño») s'oppose à celui de l'enfermement et de l'asphyxie ("puertas», «jaula»). L'association de la danse à la poésie serait ainsi un procédé permettant justement un affranchissement, une libération de la voix poétique et du corps.

Le dernier poème faisant directement allusion à la danse s'intitule "El bailarín » (Thénon, 2011a : 69). L'incipit du poème, par l'écho phonétique et par l'emploi de deux sèmes ayant la même racine, rappelle le "vivre à propos » de Montaigne et la célèbre formule «Quand je danse, je danse » (1979: 1097). La figure du danseur est vaporeuse : tous les éléments qui s'y rapportent sont éthérés ("aire», "sombra ", " humo»). Le passage d'un point de vue extérieur (troisième personne de singulier) à un point de vue interne (première personne du singulier) révèle la place centrale d'une voix poétique qui s'incarne dans la figure du danseur. La profusion d'adjectifs possessifs et de pronoms compléments (directs ou indirects) confirme la place privilégiée qu'occupe le «je » dans ce poème.

18 Le sujet danseur est le témoin du passage du temps. L'immobilité est synonyme de mort («la muerte es mi quietud»). D'où la prépondérance du mouvement, marqué par la formule tautologique «bailo todos los bailes ». Le poème adopte la forme d'une danse de l'oscillation. Les forces contraires de décomposition et de recomposition coexistent (« me deshago / y me uno »), ainsi que des métamorphoses aquatiques successives symbolisant le polymorphisme du corps du danseur (« soy mar, el hombre mar : / mi cuerpo es ola, mi mano es pez »). Sa corporalité est directement liée au rythme de la nature et aux aléas de la vie ; le sujet poétique devient pur changement, pur mouvement. Par ailleurs, l'énergie vitale exprimée à travers la métaphore maritime confère au corps dansant une puissance érotique en contraste avec l'inertie minérale, cette dernière renvoyant à la pulsion de mort (« mi dolor es piedra y sal»).

Ces poèmes montrent que la danse apparait comme horizon thématique et esthétique dès la première phase poétique de Thénon. La focalisation sur cet art permet une exploration du rapport du corps au mouvement et à l'espace. Mais cette recherche réapparaîtra de manière bien plus prononcée dans la dernière phase poétique, après l'intermède photographique. Dans distancias, les sauts et les soubresauts pulsionnels développent la dynamique libératrice d'une voix poétique qui, dans le dépouillement et dans la présence, tente de trouver son authenticité.

20 Pendant la période où Thénon se consacre davantage à la photographie qu'à la poésie, a lieu un évènement marquant. La rencontre avec la danseuse Iris Scaccheri (La Plata 
1949 - Buenos Aires 2014). Quelles résonances existent entre ce travail chorégraphique et l'imaginaire thénonien? Peut-elle jouer un rôle d'élément modificateur de l'expérience poétique? Comment se manifeste la rencontre entre la poésie et la danse? Quelles modifications induit le contact avec la danse sur l'écriture poétique?

C'est justement dans le désir, la solitude et l'organicité de la danse d'Iris Scaccheri que Susana Thénon trouve une résonance avec sa manière de voir et de représenter le monde. La danse d'Iris Scaccheri impressionne :

Deslumbraba por sus sobresalientes dotes expresivas y técnicas: los giros y los equilibrios de duraciones inverosímiles, y los saltos absolutamente ingrávidos se sostenían sobre un fluír energético -su rasgo quizá más sobresaliente-con el cual el movimiento más vigoroso podía derivar a otro delicadísimo y luego a otro de una crispación grotesca (Falcoff, 1990).

À la dimension naturelle et instinctive de sa danse s'ajoute une solide formation théorique et pratique. Plus tard, elle devient d'ailleurs professeur supérieur de danses classiques et modernes, ce qui lui permet de circuler librement d'un style à l'autre, en traversant les frontières, en dépassant les obstacles. Elle a une manière très personnelle d'habiter la danse. Elle fut, dans les années soixante, danseuse étoile au sein de l'ensemble de danse moderne du Théâtre argentin de la Plata. Son talent fut reconnu avec le succès de la présentation de Carmina Burana (musique de Carl Orff) en 1966, au Théâtre Colón de Buenos Aires. Sa participation active au sein de l'Institut Di Tella (un des épicentres de l'avant-garde artistique porteña dans les années soixante et soixante-dix) la situait comme une figure active dans la sphère de la création contemporaine. Son travail est également reconnu sur le plan international. Dans cet espace consacré à diverses expressions artistiques - théâtre, poésie, arts plastiques - elle ouvre la voie à l'expression de la danse. L'apparence aérienne, associée à la fermeté du corps et à la nudité des pieds, évoque indéfectiblement la danse d'Isadora Duncan. La danse de Scaccheri comme architecture mouvante du corps inspire Thénon et lui permet de découvrir une nouvelle voie d'exploration esthétique. Ce sera le point de départ d'un renforcement de la poétique du corps et du mouvement dans l'itinéraire créatif de Thénon. Pendant sept ans elle suivra de près le travail de la danseuse et cette démarche aboutit à la parution du livreobjet Acerca de Iris Scaccheri (1988), qui s'ouvre sur un poème cristallisant l'impact de cette rencontre :

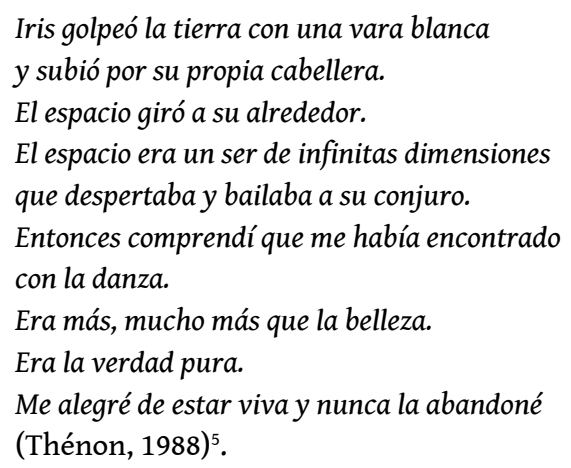

La polymétrie et la disposition graphique reflètent la souplesse des mouvements chorégraphiques. La présence de la danseuse est imposante et le mouvement descendant contraste avec le mouvement d'élévation. La personnification de l'espace opère une inversion des canons préétablis, ce qui accentue le magnétisme ensorcelant de la danseuse. La dernière partie du poème est consacrée à la découverte de la danse. L'emploi du passé simple traduit l'aspect soudain de la révélation. Cette décharge, cette secousse 
- que Deleuze dénomme la "sensation»- résulte d'une puissance libératrice qui repousse toutes les limites esthétiques pour révéler l'essence des choses.

Le dernier vers montre que la danse, dans ses aspects de jouissance, de joie et de célébration, est intégrée dans l'horizon de la poète. Valéry affirmait que la danse «se passe dans son état, elle se meut dans elle-même, il n'y a, en elle-même, aucune raison, aucune tendance propre à l'achèvement » (1957a : 1399). Au même titre que la poésie, elle n'a pas d'utilité en soi. Elle se développe au-delà du réel. Derrière leurs différences, ces deux formes artistiques partagent en outre une essence rythmique liée au corps. La structure même du poème ci-dessus évoque le mouvement chorégraphique, avec ses moments d'expansion, de rétention, de pause, de reprise : diastole et systole ; inspiration et expiration. Le rythme du poème, comme celui de la danse, se fait battement et souffle.

L'accentuation de la puissance d'Éros dans la poésie thénonienne se produit véritablement à partir de distancias. Les abîmes typographiques des poèmes cristallisent d'une certaine manière le vertige de la jouissance, d'un plaisir qui se rapproche du vertige de la mort:

Nul ne saurait nier qu'un élément essentiel de l'excitation est le sentiment de perdre pied, de chavirer. L'amour n'est pas ou il est en nous, comme la mort, un mouvement de perte rapide, glissant vite à la tragédie, et ne s'arrêtant que dans la mort. Tant il est vrai qu'entre la mort, et la " petite mort », ou le chavirement, qui enivrent, la distance est insensible (Bataille, $1988: 235$ ).

Dans distancias (1984), la poétique des espaces interstitiels atteint son apogée. Ils fonctionnent comme des écrans voilant l'accès à la complétude. Le recueil est soumis à une tension entre la fluidité produite par l'absence de ponctuation et de majuscules et l'hermétisme de ces poèmes où la matière est saturée. Le pluriel du titre souligne le caractère protéiforme de ces distances qui se déclinent au sein des trente-neuf poèmes sur les plans temporel, spatial, linguistique et ontologique. C'est en ces termes que Thénon définit l'essence de cette série poétique, dans une lettre adressée à Ana María Barrenechea datée du 17 février 1968 :

Te mando en esta carta dos poemas de la serie «nueva», los únicos hasta ahora que considero terminados. La serie se llama «distancias» y todavía no puedo explicar claramente el porqué. Sólo que tiene relación con la disociación, con la soledad, con la caducidad trágica y tierna del lenguaje, con la «distancia», aún mínima, que existe entre nosotros y nosotros mismos, o entre nosotros y lo otro (2011a : 130).

La matérialité du poème porte ainsi les traces de cette "distance ». L'alternance de la substance verbale avec les orifices, confère une dimension organique aux poèmes. Comme si l'énergie de la danse permettait une manifestation du corps plus intense. La formulation d'Alice Godfroy à propos de la relation entre danse et poésie est tout à fait éclairante car elle souligne justement le «lien nécessaire et invisible qui se joue dans l'expérience des corps » $(2014: 264)$.

Les quatre premiers vers du poème $n^{\circ} 9$ me semblent bien révéler le processus d'érotisation progressive du langage poétique en lien avec la rencontre de la danse :

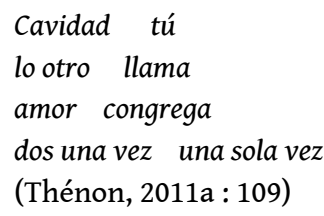

29 La fragmentation rythmique, accentuée par l'injection de vides typographiques, crée un tissu poétique fait de contractions et d'extensions, à l'image du rythme corporel de la 
respiration, du battement, de la danse. La «cavidad » et la perforation du poème par les blancs typographiques évoquent la dimension réceptive du corps féminin. Le poème fonctionne comme une chorégraphie impulsée par l'énergie d'Eros. Les blancs typographiques seraient des équivalents graphiques du saut du corps qui danse. Ils permettraient de dire l'indicible tout comme le danseur rend visible l'invisible.

À la perforation du langage poétique s'ajoute un autre procédé graphique : la mise entre parenthèses de certains mots. Les parenthèses ne se limitent pas à une fonction typographique. Elles fonctionnent comme des sutures, comme des membranes graphiques révélatrices de l'organicité du poème :

el viento hidraba (flujos) acuérdate

de sol velarios me impiden

31 Mais elles représentent graphiquement ce « lieu de l'intervalle » qu'occupe le désir selon Luce Irigaray (1984). En effet, il ne peut être fixé car «lui assigner une définition permanente revient à le supprimer comme désir ». Le désir impose donc une extrême mobilité car « désirer exige un attrait : la modification de l'intervalle, le déplacement du sujet ou de l'objet dans leurs rapports de proximité ou d'éloignement» (Irigaray, 1984 :15).

Au contact de la danse libre d'Iris Scaccheri, Thénon aurait mieux pris conscience du rythme et de la spatialité du corps, et de la nécessité de se libérer de tout cadre préétabli. Dans sa capacité à incarner des forces contraires le danseur peut incarner toutes les formes, toutes les forces. À travers le mouvement il dépasse les limites de son individualité. Il entre en contact avec la profondeur de son être pour en faire don :

Intouchable et cependant touchant, il se sert de lui-même et de la part la plus intime de lui-même - «la chair " selon Merleau-Ponty - au bénéfice de deux autres : l'œuvre et le spectateur. Il intervient non de vive voix, mais de vif corps (Dupuy, 2007 : 16-17).

Il est donc intéressant d'étudier les effets de la rencontre de la poésie et de la danse et de voir dans quelle mesure le mouvement, les sauts, et les plis du corps seraient transposés sur le corps du poème. Autrement dit, quel est le processus de résonnance entre le poème et la danse et quels sont ses effets?

34 La réflexion d'Alice Godfroy sur la correspondance entre les arts octroie une place centrale à la danse comme pratique corporelle, dont le caractère éphémère permettrait de penser le rapport à la création depuis une nouvelle perspective :

Parce qu'elle assume l'éphémère, la danse, - cancre de l'esthétique traditionnelle - serait à même de révéler le mode de phénoménalisation de toute relation à l'art, à savoir un mouvement qui exhausse une apparition, laquelle se suspend à sa propre extinction. Prendre la danse au sérieux, c'est aussi voir que, par-delà les distinctions de médium et la diversité des formes produites, les arts se conjoignent nécessairement dans l'expérience des corps et le mouvement de leur contact (Godfroy, $2014: 271$ ).

Le corps serait donc le point de convergence des expressions artistiques. Nous verrons dans la partie suivante que le corps a pris une place centrale dans la poésie de Thénon à la suite de la rencontre de cette dernière avec la danse. 


\section{Entrelacs : une poésie en danse} part parce que ce recueil est le fruit d'une mûre et longue réflexion (seize ans s'écoulent entre sa conception et sa publication). D'autre part parce qu'il est chargé d'une véritable force transgressive. Le titre même ouvre deux lectures possibles : la première souligne l'importance thématique de l'écart, de la disjonction, de la séparation. La deuxième opère au niveau formel. La distance signifie l'éloignement des canons lyriques. Mais en tant que pilier de la poétique de Thénon, elle devient aussi un procédé formel à travers lequel il est possible de proposer un langage expérimental, en mouvement.

L'injection de blancs typographiques, de respirations, ne rend pas les poèmes plus légers. $\mathrm{Au}$ contraire, cette matière blanche faite de vide renforce leur épaisseur. Cette densité les rend hermétiques. C'est pourquoi ils incitent, comme le souligne Ana María Barrenechea, à une pratique de lecture de l'effort presque physique :

Su conjunto funda un espacio que, paradójicamente, se abre en líneas de fuga y al mismo tiempo se cierra en circularidad angustiante. Fragmenta, quiebra, desgarra, y a la vez organiza una constelación poética que se revela al lector por su pura presencia, sorprendente $e$ inevitable. Su extrañeza no impide la comunicación. Todo lo contrario. Lo que hace es pedirla, incitarla. El libro, con la variedad de voces que en él dialogan, dice al lector sin descanso: busca, soy múltiple, no te detengas, no te conformes (Thénon, 2011a : 130).

Le sujet poétique de distancias mute en permanence. Il est pluriel, dissocié et fractionné, à l'image du corps dansant qui décompose le mouvement :

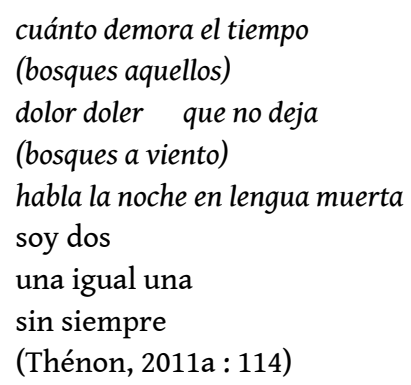

La polymétrie des trois derniers vers (2/5/3) met l'accent sur le morcellement d'un sujet poétique. L'essence contradictoire et mobile de ce dernier rappelle celle du danseur. Les blancs typographiques renvoient à une segmentation du sujet mais aussi à la rétention du souffle qu'effectue le danseur qui saute :

Lorsque le danseur décolle, il chasse le courant du souffle comme un éclair qui le parcourt de bas en haut, des pieds à la tête, afin de retenir son souffle depuis le moment où il quitte le sol jusqu'à l'apogée du saut, et au-delà. Pendant ces quelques secondes d'effort intense, retenant son souffle, il défie effectivement la gravité, devient créature aérienne et semble voler ou flotter à travers l'espace. C'est seulement dans la courbe de la retombée que le souffle retrouve le corps qui se détend et renvoie le danseur vers la terre après un bref envol (Wigman, 2011 : 127).

Le paratexte même démontre que l'idée de mouvement est centrale dans ce recueil placé sous le signe de la danse. Celle-ci est exposée dès la couverture du livre (dans la première édition de 1984), avec une photographie en noir et blanc d'Iris Scaccheri, réalisée par Susana Thénon. La danseuse apparaît de profil entre deux lignes blanches parallèles. Le corps est sur le point de s'élever. Cette impression est soulignée par la position des bras, tendus parallèlement vers l'arrière. La photographie fige un instant qui se situe entre le 
moment de l'impulsion, de l'élan et celui de l'élévation, du saut. Elle illustre un entredeux. Le parallélisme des lignes blanches - qui évoquent une échelle dont on ne voit pas les marches - fait écho à l'écart entre les bras et les pieds.

Ce paratexte situe d'entrée le recueil sous le signe de la danse et de l'écart. Cela est d'ailleurs confirmé par les remerciements que la poète adresse à Iris Scaccheri :

Y muy en especial a Iris Scaccheri, quien sin saberlo me infundió con su danza la visión definitiva para reelaborar y completar esta obra, cuya duración lindaba peligrosamente con lo interminable (Thénon, 2011a : 101).

La structure de distancias est circulaire puisque le premier et le dernier poème sont identiques. Le recueil est habité par la tension entre l'inertie et le mouvement, la substance et le vide. La voix poétique adopte la forme d'un écho. Cette circularité est soulignée par le motif de la roue développé au sein de ce poème-miroir :

la rueda se ha detenido se ha deteni-

dos tres dos tres dos la rueda

se ha detenido roto por dentro

La colonne vertébrale de ce poème-miroir est hélicoïdale: elle se compose de blancs typographiques et vient troubler le mouvement linéaire de la lecture occidentale conventionnelle. Ce poème inaugural vise à adapter la vue du lecteur au relief sismique de ces poèmes, au sein desquels la matière du verbe poétique est aussi importante que ses failles. Ce vide coagulé - comme le saut en danse - dit quelque chose. Les mots sont confrontés en permanence à un abîme. Le verbe poétique se construit par à-coups répétitifs. Il est contraction et spasme. Ces convulsions poétiques composent une structure proche du pli et du repli deleuziens. Les flexions forgent également des espaces tautologiques qui dessinent le mouvement de l'éternel retour du même.

Pour illustrer ce rapport étroit entre danse et poésie j'analyserai ici les trois poèmes qui sont, à mon sens, les plus significatifs $\left(n^{\circ} 2, n^{\circ} 7, n^{\circ} 20\right)$. Le premier $\left(n^{\circ} 2\right)$ offre une vision du monde similaire à une chorégraphie de la distance.

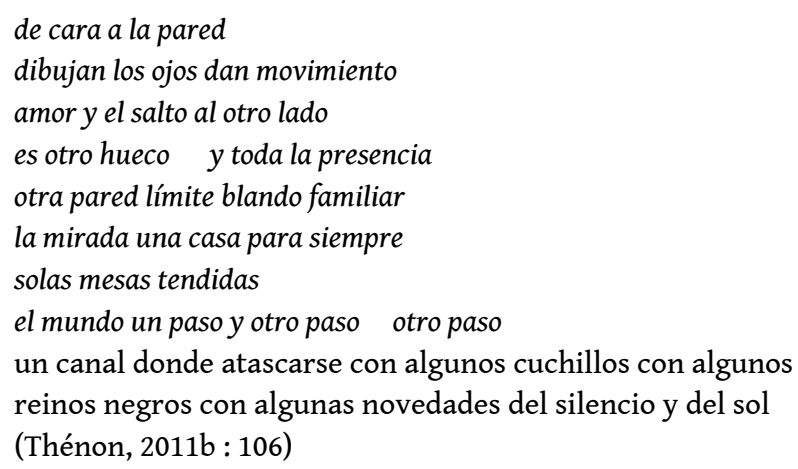

L'écart est symbolisé par les murs (" pared », « otra pared »). En espagnol, l'anagramme de «pared » est " padre ». Si l'on met cela en rapport avec les catégories du symbolique (loi du père) et la sémiotique (loi de la mère) développées par Kristeva (1974) - autour desquelles on peut articuler la réflexion sur l'ouverture progressive du langage poétique de Thénon -, on voit apparaître au sein du poème une zone conflictuelle. Le sujet poétique se heurte aux limites sociales et linguistiques. Mais il recherche une zone d'expression pulsionnelle, représentée ici par les dessins de mouvement qui rappellent la composition de la danse ("dibujan los ojos dan movimiento»), par les sauts et le dépassement des frontières («amor y el salto al otro lado»; «otra pared límite blanco familiar »). L'introduction de blancs typographiques, ainsi que la mention du vide («es 
otro hueco y toda la presencia ») et du pas (« un mundo, un paso y otro paso otro paso ») développent la problématique du surgissement d'obstacles et de leur dépassement. Ces deux éléments résonnent fortement avec le danseur comme figure capable d'incarner la dislocation, selon la formule de Georges Didi-Hubermann :

Aussi peut-on imaginer que le danseur, sur scène, se pare de la beauté de ses gestes, s'engendre lui-même en posant la question du désir et, puisque désir il y a, ne danse qu'à se séparer, par brisures et par "intervalles qui se répètent ». Voilà pourquoi il est solitaire et partenaire en même temps. C'est en tant qu'il se sépare - de l'objet qu'il vise, de lui-même et, enfin, de nous, ses spectateurs - qu'il nous concerne si intimement, offrant figures et mouvements pour nos propres solitudes (2006: 169).

Le poème devient l'espace d'exposition de ce conflit entre la linéarité monotone du langage et sa libération, son explosion, son atomisation à travers des blancs qui redistribuent le silence sur la masse poétique, tout comme les arrêts du danseur rythment le mouvement et annoncent de nouvelles figures. Dans ce poème, le silence, associé à l'absence de mouvement, ne plonge pas le sujet dans une inertie malsaine. Au contraire, à partir de ces vides, le sujet et le verbe poétique peuvent se renouveler, comme l'atteste la représentation d'une autre voix en italique qui souligne la fécondité du silence (" novedades del silencio y del sol »). Les pas et les capsules de vide libèrent le langage de sa linéarité et le ramènent à son expression la plus organique, la plus directe, la plus pulsionnelle.

Être dans le mouvement, c'est être hors des choses, hors des cadres habituels, où les choses se distribuent avec plus ou moins de stabilité dans l'espace. Si le danseur produit « une forme de temps », comme l'écrit Valéry, cette forme ne sera liée qu'à «l'infinité de ces nobles similitudes, les conversions, les inversions, les diversions inépuisables qui se répondent et se déduisent sous nos yeux " (1957b: 155). La danse comme " acte pur des métamorphoses » (1957b : 165) fait éclater toute unité du corps. «Ce Un veut jouer à Tout. [...] Il veut remédier à son identité par le nombre de ses actes! Étant chose, il éclate en événements !» (1957b : 171).

Le poème $\mathrm{n}^{\circ} 7$ prolonge ce même mécanisme de fractionnement qui s'avère toutefois plus complexe, puisqu'il met en place une dimension polyphonique. Ainsi on est face à un système de dédoublement de la voix, d'échos, signifié textuellement par la différenciation typographique et auditivement par les variantes tonales employées.

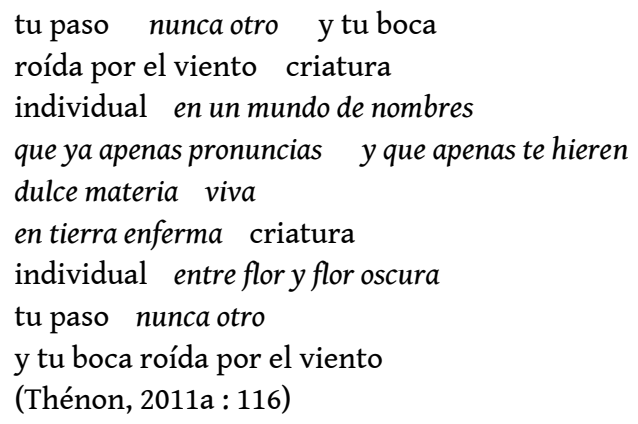

49 Je ne développerai pas ici la dimension polyphonique de la poétique thénonienne. En revanche, il me semble important d'analyser la poétique du pas et du saut telle qu'elle apparait dans cette série. On peut diviser le poème en deux parties. La première couvre les quatre premiers vers. La seconde va du sixième au neuvième vers. Le cinquième vers («dulce materia viva ») situe la corporalité comme axe du poème. Derrière ce découpage, la répétition de certains mots ou fragments produit un effet de circularité : 
«tu paso " (premier et huitième vers) ; «criatura / individual » (deuxième et troisième vers, septième et huitième vers).

Un fragment des premier et deuxième vers subit par ailleurs une variation, pour apparaître comme une unité au dernier vers: "y tu boca roída por el viento ». Ce processus de défragmentation révèle néanmoins des variations et des différences dans ce poème hétérogène.

Dans le poème $\mathrm{n}^{\circ} 20$ de distancias, c'est un sujet poétique dévoré par la passion amoureuse qui est représenté. L'image ignée symbolise cette passion destructrice. L'emploi du passé simple signifie la séparation, alors que les actions se succèdent. Ce qui m'intéresse particulièrement ici, c'est la coexistence des forces contraires, révélatrice du rapport conflictuel du sujet poétique au monde. Ce poème est chargé d'une force chorégraphique, dans le sens où il s'articule autour du mouvement du présent vers le passé ( de un tiempo en retroceso »), qui crée une dynamique cyclique (« morí / para nacer »). Le sujet supporte et porte l'autre («te alzaban / mis hombros ») au milieu d'une situation proche de l'hallucination (« sí temblor carnívoro »). Ce mouvement d'élévation contraste avec la manifestation d'autrui, dont la présence est révélée par le bruit des pas sur le sol. L'amalgame entre les lettres, les mots d'autrui et les pas est signe de présence. La fermeture du poème sur le mot "pasos" révèle l'importance des pas - vecteurs de déplacement - dans la poétique de Thénon. La topographie poétique (parsemée de blancs, d'arrêts, de reprises) est semblable à un tracé chorégraphique, avec la dose de fluctuation que cela implique nécessairement. Cela confère une dimension particulière à la matérialité du poème.

Dans les poèmes composés par Thénon dans les années quatre-vingts, le sujet poétique cherche un espace, un ailleurs. Les blancs typographiques sont des marques concrètes de ce déplacement permanent. Ils créent une écriture du saut et du sursaut. Mais, lorsque ce besoin ne se manifeste pas au niveau de la matérialité du poème, il apparaît évoqué au niveau sémantique. Plusieurs poèmes formulent le désir de voler, de s'envoler :

repta desesperado cielo abajo
cariátide en los altos
jirón de cable
tiempo ilegible
la trama está hilada
cómo alzaré vuelo
todo este cuerpo es presa
de la gravedad
otro ser es un nombre
otro ser es su espacio
el miedo es la ventana
donde no asoman
(Thénon, 2011b : 82)

La danse répond à l'interrogation du poème. Elle permet d'aller au-delà de soi-même, pour devenir autre et atteindre ainsi un autre espace. L'image du fil (« cable », « hilada ») et de la suspension («altos», "alzaré vuelo») évoquent la figure du sujet poétique comme danseur-funambule, décrite par Jean-Michel Maulpoix :

Qu'est-ce donc que le poème, sinon une affaire de trame et de filage, avec des mots "tirés de soi(e)»: le fil horizontal des vers croise le fil vertical des rimes. Le vers est l'en allée, la solitude de la phrase. La rime est le retour, le mouvement de navette, le nœud de l'identité. Le poème inscrit jusque dans sa forme la fièvre du départ, le désir de l'envol, et le principe de réalité avec lesquels ces aspirations 
doivent composer, jusqu'à produire un objet dansant et pensant qui cadastre, ajointe, relie et prend la mesure juste du désir et de son défaut (1998). soumis aux forces du devenir :

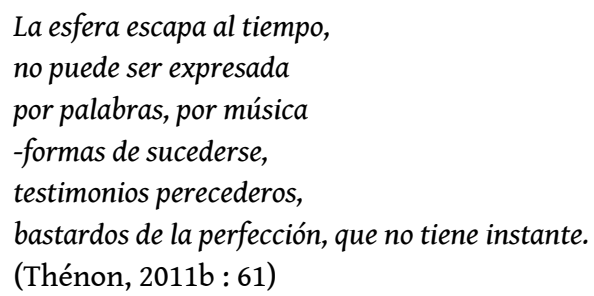

59 Associée à la poésie, la danse permet de canaliser des questionnements sur l'être-aumonde depuis la perspective de la corporalité, qui devient dans le poème «corps- 
oralité ». Par la danse, le mouvement s'infiltre dans sa poétique et met en branle la matérialité poétique.

En effet, Susana Thénon remet en question le fonctionnement et les codes de la poésie et du langage. Sous sa plume, le verbe poétique subit plusieurs métamorphoses. Il devient matériau malléable, susceptible de succomber lui aussi à l'ivresse dionysiaque. Certains poèmes inédits, tel «Crónicas del sur de la sangre VIII» (2011b : 48), confirment l'intérêt qu'elle portait dès ses premiers poèmes au langage onomatopéique :

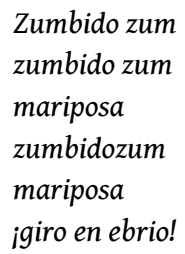

Le langage vidé de son sens devient pur son, pur mouvement du visible, pur geste ivre. Le projet de Thénon s'inscrit dans une logique subversive et libératrice qui cherche à rompre avec la linéarité. Casser les schémas préétablis implique nécessairement l'éclatement du langage. Un des poèmes d'Habitante de la nada, «Caos» (2011a: 61), est déjà placé sous le signe de la révolte, même si celle-ci ne s'y exprime pas encore au niveau formel. On voit par là que la poétique de Thénon est profondément connectée avec la liberté de la danse. Avancer en ligne droite, sans bifurcation, sans imprévus, conduit à la monotonie d'une existence prévisible et coercitive. Les tournures privatives ( no tienen más que avanzar »; " no tienen más alternativa que delante ») et négatives ("Son señales de nada »; " no ayuda »; « no invita ») reflètent la stérilité d'un itinéraire existentiel de ce type. Le dernier vers fonctionne comme art poétique («no invita al caos preparado como una fiesta »). La négation ne place pas le chaos dans la sphère des interdits. Bien au contraire, elle suggère que le chaos, le mouvement, l'excès doivent les remplacer. Créer une écriture " poétichorégraphique » serait une manière d'avancer vers la liberté.

La richesse expressive et l'originalité de l'œuvre de Thénon est confirmée par la circulation artistique qui s'établit entre la poésie et la danse.

On distingue deux moments dans la relation de la poésie et de la danse dans ce processus créatif. Le premier touche ses recueils initiaux : même si l'approche est timide, l'inclusion de poèmes construits autour de la danse et de la figure du danseur permet de mieux dessiner les contours d'un corps en mouvement, afin d'ouvrir un nouvel horizon esthétique pour son écriture postérieure (entre 1968 et 1988). Le contact entre poésie et danse relevant d'une dimension thématique ou métaphorique cède alors la place à une véritable circulation entre ces deux arts. Une telle évolution témoigne d'un processus de libération. Mais aussi d'une intégration du rythme et du mouvement du corps en danse comme matériau poétique.

64 La poétique de Thénon peut parfaitement s'inscrire dans la mouvance décrite par de récents travaux sur la correspondance des arts qui confèrent au corps une place centrale dans le rapport entre danse et poésie (Finck, 1992; Godfroy, 2014). Comme le souligne très justement Alice Godfroy, « les arts communiquent nécessairement dans l'expérience des corps » $(2014: 271)$.

C'est effectivement la corporalité qui matérialise, chez Thénon, le lien entre danse et poésie. On a vu comment s'est progressivement établi un parallèle entre l'acte d'écriture et le geste de danse, non seulement au niveau thématique mais aussi au niveau de la matérialité même du poème. La rencontre avec la danse - personnelle, vraie et 
organique - d'Iris Scaccheri fut fondamentale pour Susana Thénon. Le contact avec la danse contemporaine est libérateur car, comme le rappelle Michèle Fink :

L'acte fondateur de la danse au vingtième siècle est justement la mise en crise d'une conception de la danse comme art poétique de la métaphore (Martha Graham). La danse n'est plus une poétique qui vise l'évasion du corps vers une signification allégorique, mais une poétique qui fonde son espoir sur l'identification du corps et de la vérité : « Le mouvement ne ment pas » (1992).

C'est dans la fragmentation, l'ambigüité, la fugacité, l'action et la contraction du corps en danse que Thénon semble trouver une possibilité d'ouverture. La liberté de la danse lui apporte une énergie supplémentaire, permettant de rompre avec les schémas poétiques préétablis.

67 La dislocation du langage, qui fait primer la littéralité et la matérialité sur la référentialité, aboutit à une écriture en mouvement et du mouvement. Quand la poésie embrasse la danse, le mot, le vide, l'inexprimable (voire l'innommable) ${ }^{7}$ deviennent ainsi les doubles du pas, du saut, de l'invisible. Les perforations, les sutures textuelles cristallisent une dimension organique du poème et soulignent l'aspect mobile - voire physique - de leur écriture et de leur lecture. À propos de distancias, Ana María Barrenechea souligne qu'il s'agit d'une :

Experiencia textual única por la complejidad de relaciones simultáneas que establece en todas las direcciones y en todos los niveles: red móvil engendrada dentro de cada poema y por la sucesión de los poemas, siempre en proceso (Thénon, 2011: 130).

Ces fissures textuelles permettent l'infiltration du corps et de la danse car elles constituent aussi bien des silences, des respirations, des sauts, des pas, des abîmes. Elles annoncent par leur craquellement une éclosion vers un art total à l'intérieur duquel les frontières se dissolvent.

\section{BIBLIOGRAPHIE}

ANZIEU Didier (1995), Le Moi-peau, Paris : Dunod.

BATAILLE Georges (1988), Euvres complètes, Paris : Gallimard.

CIFUENTES Juanita (2010), Les Métamorphoses de la voix poétique dans l'œuvre de Susana Thénon, thèse de doctorat en Études hispaniques et hispano-américaines, Université de Grenoble.

CORTÉS ROCCA Paola (2013), "Rueda de mujeres. Acerca de Susana Thénon", Cuadernos Lírico, 9, en ligne sur <http://lirico.revues.org/1125>, ( $1^{\mathrm{er}}$ septembre 2013).

DI CIó Mariana (2003), “La cara deshecha en el vidrio cuarteado”. La identidad del sujeto poético en Edad sin tregua de Susana Thénon (mémoire de master 2 non publié), Universidad Católica de Buenos Aires, Argentine.

Di Ció Mariana (2004), “La 'quebrada geometría’ de Edad sin tregua de Susana Thénon”, Espéculo. Revista de Estudios Literarios.

DIDI-HUBERMANN Georges (2006), Le danseur de solitudes, Paris : Minuit. 
DUPUY Dominique (2007), Danse contemporaine, pratique et théorie, Marseille : Images en manœuvres / Le Mas de la Danse.

FALCOFf Laura (1990), “Scaccheri en el San Martín”, Página 12.

FINCK Michèle (1992), « Poésie moderne et danse : le corps en question », Poésie et danse à l'époque moderne. Corps provisoire, en ligne sur le site de Michèle Finck : <http://michele.finck.free.fr/ poesie_moderne_et_danse.htm>.

GODFROY Alice (2014), « De la nécessité d'une correspondance entre les arts : la danse révélatrice », Y. M. Ergal \& M. Finck (dir.), Littérature comparée et correspondance des arts, Strasbourg : Presses universitaires de Strasbourg.

IRIGARAY Luce (1984), Éthique de la différence sexuelle, Paris : Minuit.

KRISTEVA Julia (1974), La révolution du langage poétique, Paris : Seuil.

NIETZSCHE Friedrich (1903), Euvres complète. Vol. 9 : Ainsi parlait Zarathoustra (H. Albert, trad.), Paris : Société du Mercure de France.

MANZANO Inés (2009), « Loba Completa », Revista Lamás médula, en ligne sur <http://

www.revistalamasmedula.com.ar/nro2/nota1.htm>.

MAULPOIX Jean-Michel (1998), « Le danseur de corde portait du poète en funambule ", Nouveau recueil (46), dossier « Figures du poète », en ligne sur <http://www.maulpoix.net/danseur.html> (également publié dans MAUlPoIx Jean-Michel (2004), Adieux au poème, Paris : José Corti).

MONTAIGNE (de) Michel (1979), Les Essais, Livre III, Paris : Flammarion.

SCACCHERI Iris (2011), Brindis a la danza, Buenos Aires : Paidós.

THÉNON Susana (1958), Edad sin tregua, Buenos Aires : Cooperativa Impresora y Distribuidora Argentina Ltda.

THÉNON Susana (1959), Habitante de la Nada, Buenos Aires : Thiriel. Editora e Impresora Americalee.

THÉNON Susana (1967), De lugares extraños, Buenos Aires : Carmina.

THÉNON Susana (1968a), « “Dos poetas” ensayos sobre Juan Luis Morabes y Rodolfo Benasso », Sur (312).

THÉNON Susana (1968b), « Oliverio Girondo: una historia del fervor », Sur (315).

THÉNON Susana (1979), Rainer María Rilke. Palabra e imagen, Buenos Aires : Instituto Goethe.

THÉNON Susana (1984), distancias, Buenos Aires : Torres Agüero.

THÉNON Susana (1987), Ova completa, Buenos Aires : Suramericana.

THÉNON Susana (1994), distancias/distances, Los Angeles : Sun \& Moon Press, édition bilingue espagnol-anglais (R. Treitel, trad.).

THÉNON Susana (1998), Acerca de Iris Scaccheri, Buenos Aires : Anzilotti.

THÉNON Susana (2011a), Susana Thénon. La Morada imposible, tome 1, Buenos Aires : Corregidor (textes réunis et commentés par A. M. Barrenechea \& M. Negroni).

THÉNON Susana (2011b), Susana Thénon. La Morada imposible, tome 2, Buenos Aires : Corregidor (textes réunis et commentés par A. M. Barrenechea \& M. Negroni).

TIRRI Néstor (2000), « El perenne fuego sagrado de Iris », La Nación (8 décembre 2000). 
VALÉRY Paul (1957a), « Philosophie de la danse », CEuvres, tome 1, Paris : Gallimard.

VALÉRY Paul (1957b), « L'âme et la danse », CEuvres, tome 2, Paris : Gallimard.

WIGMAN Mary (2011), « Le langage de la danse », C. Macel \& E. Lavigne (dir.), Danser sa vie - Écrits sur la danse, Paris : Éditions du Centre Pompidou.

\section{NOTES}

1. Thénon traduit une sélection de poèmes de Rilke, publie des essais dans les revues Ficción et Sur, expose une série de photographies inspirées des poèmes de Rilke à l'institut Goethe en 1979, expose un ensemble de soixante photographies au Centre d'Art et de Communication de Buenos Aires en 1982, dont la quatrième et dernière série intitulée "Los reales espejismos » est consacrée à la danseuse Iris Scaccheri. En 1988 un livre-objet intitulé Acerca de Iris Scaccheri rassemble 36 clichés portant spécifiquement sur la danseuse argentine.

2. Je limite ici mon corpus d'étude au rapport entre danse et poésie. Pour une analyse intégrant également la dimension photographique de l'œuvre de Susana Thénon, voir Cifuentes (2010) et Cortés Rocca (2013).

3. Leonor Vassena est une figure importante du mouvement naif en argentine. Elle et son mari, l'écrivain Alberto Girri, furent, dans les années 1950, des personnalités actives du monde culturel argentin.

4. Sur la dimension lyrique des premiers poèmes de Thénon, voir Di Ció (2003) et Cifuentes (2010).

5. Thénon photographia des moments de Carmina Burana: Yo odio, yo amo, La muñeca, Juana, reina de Castilla y Aragón, Hosanna, ¿Me quisite alguna vez?, Homenaje a Dore Hoyer, Temas españoles, La ascensión et Dos mujeres.

6. Elle définissait la danse en ces termes : "Tomar ese cuerpo y llevarlo al universo de lo que se ve y de lo que no se ve. Lo que se ve: ser estrella, ser pájaro, ser gato, ser árbol, ser dolor, ser amor. Lo que no se ve: lo que es más que la estrella, que el pájaro, que el árbol. Y cuando los que observan esto quedan transformados (ellos también) en flor, en pájaro, y olvidan que el medio es un cuerpo con brazos y dedos, en ese instante se produce la danza » (Tirri, 2000).

7. Le recueil distancias a été publié en 1984, juste après le retour de la démocratie en Argentine et après plus de sept ans d'une dictature militaire sanglante.

\section{RÉSUMÉS}

L'itinéraire poétique de Susana Thénon (Buenos Aires 1937-1990) est marqué par la circulation artistique entre la poésie, la musique, la photographie et la danse. Dans cet article je m'intéresse spécifiquement à la relation entre la poésie et la danse dans l'œuvre de cette poétesse argentine. Dans une première partie j'observe dans quelle mesure il s'agit d'une poésie sur la danse, et comment ces deux arts entrent en contact pour analyser ensuite leur mécanisme d'entrelacement.

The poetic itinerary of Susana Thénon (Buenos Aires 1937-1990) is marked by the artistic movement between poetry, music, photography and dance. In this article I'm specifically 
interested in the relationship between poetry and dance in the work of this Argentine poet. In the first part I see how this is a poetry about dance and how these two arts come into contact and then I analyze their interlacing mechanisms.

El itinerario poético de Susana Thénon (Buenos Aires 1937-1990) está marcado por la circulación artística entre la poesía, la música, la fotografía y la danza. En este artículo me intereso específicamente en la relación entre la poesía y la danza en la obra de esta poetisa argentina. En un primer momento observo en qué medida se trata de una poesía sobre la danza y cómo entran en contacto estos dos artes, para analizar luego los mecanismos de entrelazamiento.

\section{INDEX}

Mots-clés : Susana Thénon, poésie, danse, circulation entre les arts

Keywords : Susana Thénon, poetry, dance, artistic circulation

Palabras claves : Susana Thénon, poesía, danza, circulación artística

\section{AUTEUR}

\section{JUANITA CIFUENTES-LOUAULT}

Univ. Grenoble Alpes [ILCEA4], F - 38040 Grenoble

Note biographique : Juanita Cifuentes-Louault est titulaire d'un doctorat en Études hispaniques et hispano-américaines (Université de Grenoble, 2010). Actuellement professeur agrégée d'espagnol au Lycée français Jean-Monnet (Bruxelles), elle est également chercheure à l'Université Grenoble Alpes (ILCEA4-CERHIUS) et collaboratrice scientifique à l'Université catholique de Louvain (centre ECR-INCAL). 